\title{
COMBINED SURFACE TENSION AND BUOYANCY- DRIVEN CONVECTION IN A RECTANGULAR OPEN CAVITY IN THE PRESENCE OF A MAGNETIC FIELD
}

\author{
N. Rudraiah \\ Gulbarga University, Gulbarga 585 106, India \\ and \\ M. Venkatachalappa* and C. K. Subbaraya \\ UGC-DSA Centre in Fluid Mechanics, Department of Mathematics, Central College Campus, \\ Bangalore University, Bangalore 560001 , India
}

(Received 12 February 1994; in revised form 10 April 1995)

\begin{abstract}
A numerical study is conducted to understand the effect of magnetic field on the flow driven by the combined mechanism of buoyancy and thermocapillarity in a rectangular open cavity filled with a low Prandtl number fluid $(\operatorname{Pr}=0.054)$. The two side walls are maintained at uniform but different temperatures $\theta_{h}$ and $\theta_{c}\left(\theta_{h}>\theta_{c}\right)$, while the horizontal top and bottom walls are adiabatic. A finite difference scheme consisting of the ADI (Alternating Direction Implicit) method, which incorporates upwind differencing for non-linear convective terms and the SLOR (Successive Line Over Relaxation) method are used to solve the coupled non-linear governing equations. Computations are carried out for a wide range of Grashof number $G r$ ranging from $2 \times 10^{4}$ to $2 \times 10^{6}$, Marangoni number $\mathrm{Ma}$ from $\mathrm{O}$ to $10^{5}$ and Hartmann number $\mathrm{Ha}$ from 0 to 100 . The detailed flow structure and the associated heat transfer characteristics inside the cavity are presented. At large $M a$, two counter-rotating cells are formed at the upper half and lower half of the enclosure. As $\mathrm{Ha}$ increases, the temperature field resembles that of a conduction type and the streamlines are elongated in nature in the horizontal direction. The upper cell is crowded and stretched along the free surface. The average Nusselt number increases with $M a$ but decreases with $\mathrm{Ha}$.
\end{abstract}

\section{INTRODUCTION}

It is well known that buoyancy forces can induce motion in liquids contained in enclosures with differentially heated side walls. In addition, when a free liquid surface is present, variations in the liquid surface tension at the free surface-due to temperature gradients can also induce motion within the fluid called thermocapillary flows (Marangoni convection). The flows associated with these two mechanisms, considered independently, have been extensively examined by many authors. Recently, considerable interest has been generated on the interaction of buoyancy and surface tension driven convection, motivated primarily by the concerns of material processing technology. Combined buoyancy and thermocapillary convection in a differentially heated cavity has been considered by Bergman and Ramadhyani [1]. They conducted a numerical study of steady convective flow in a square cavity and showed that surface tension effects can significantly alter the buoyancyinduced flow. Srinivasan and Basu [2] numerically computed the thermocapillary flow in a rectangular cavity during laser melting. The gas-liquid interface was assumed to be flat with sinusoidal variation of temperature. Thereafter, Basu and Srinivasan [3] numerically simulated a two-dimensional steady-state laser melting problem in a cavity, whereas Chen and Huang [4] conducted a similar study with a moving heat flux. Carpenter and Homsy [5] studied the problem of combined buoyancy-thermocapillary convection in a cavity with a free surface heated differentially in the horizontal direction. The boundary layer scalings for buoyant and thermocapillary convection have been well established. More

* Author to whom future correspondence should be addressed.

Contributed by K. R. Rajagopal. 
recently, Ben Hadid and Roux [6] have studied numerically the influence of thermocapillary forces on buoyancy-driven convection for a shallow open cavity with differentially heated end walls and have shown that thermocapillarity can have a quite significant effect on the stability of a primary buoyancy-driven flow.

Most studies found in the literature on combined surface tension and buoyancy-driven convection in a rectangular enclosure are concerned with electrically non-conducting fluids. Attention has not been given to the study of combined buoyancy and surface tension driven convection of an electrically conducting fluid in the presence of a magnetic field in a rectangular enclosure, in spite of its important applications in crystal growth techniques (for example, Czochralski and Bridgman techniques). Convection is an important phenomenon in these techniques as it can account for heat transfer in the liquid phase and can change material physical properties. Studies have shown that in order to enhance crystal purity, increase compositional uniformity, and reduce defect density, it is necessary to control the transport processes. These unavoidable hydrodynamic movements can be damped with the help of a magnetic field. Thus the present numerical study investigates the effect of magnetic field on the combined mechanism of thermocapillary and buoyancydriven convection in a rectangular cavity filled with a low Prandtl number fluid $(\operatorname{Pr}=0.054)$. Calculations are carried out by solving two-dimensional Navier-Stokes equations, coupled with energy equation, for Grashof number $(G r)$ ranging from $2 \times 10^{4}$ to $2 \times 10^{6}$, Marangoni number $(\mathrm{Ma})$ from 0 to $10^{5}$ and Hartmann number $(\mathrm{Ha})$ from 0 to 100 . The detailed flow structure and the associated heat transfer characteristics inside the cavity are presented.

\section{MATHEMATICAL FORMULATION}

Consider a two-dimensional rectangular open cavity of width $L$ and height $H$ as shown in Fig. 1. The vertical walls located at $y=0$ and $y=L$ are isothermal but at different temperatures $\theta_{c}$ and $\theta_{h}$, respectively. The bottom and top of the enclosure is assumed to be thermally insulated. The cavity is filled with a low Prandtl number fluid (liquid metal) which is electrically conducting. The Cartesian coordinates $(x, y)$, with the corresponding velocity components $(u, v)$, are as indicated in the figure. The gravity acts in the $x$-direction. The upper free surface is assumed to be flat and non-deformable. This assumption is reasonable for liquid metals as their interfacial tensions have relatively large values and $90^{\circ}$ contact

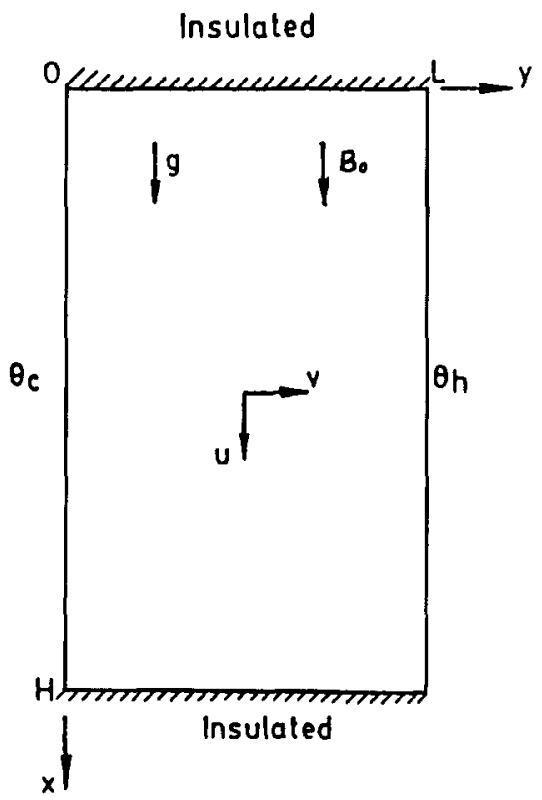

Fig. 1. Schematic of the physical system. The upper surface is free. 
angles between the vertical solid wall and the meniscus. The external magnetic field is applied parallel to the gravity. The induced magnetic field is negligible compared to the applied magnetic field. The surface tension on the upper boundary is assumed to vary linearly with temperature; that is,

$$
\sigma=\sigma_{0}\left[1-\gamma\left(\theta-\theta_{0}\right)\right],
$$

where $\gamma=-1 / \sigma_{0}(\partial \sigma / \partial \theta)$ is the temperature coefficient of surface tension and the subscript 0 refers to a reference state. The equations governing the laminar two-dimensional flow of a Newtonian, electrically conducting, Bousinesq fluid may be written as

$$
\begin{aligned}
& \frac{\partial u}{\partial x}+\frac{\partial v}{\partial y}=0 \\
& \frac{\partial u}{\partial t}+u \frac{\partial u}{\partial x}+v \frac{\partial u}{\partial y}=-\frac{1}{\rho_{0}} \frac{\partial p}{\partial x}+v \nabla^{2} u-g \beta_{T}\left(\theta-\theta_{0}\right) \\
& \frac{\partial v}{\partial t}+u \frac{\partial v}{\partial x}+v \frac{\partial v}{\partial y}=-\frac{1}{\rho_{0}} \frac{\partial p}{\partial y}+v \nabla^{2} v-\frac{\sigma_{e} B_{0}^{2}}{\rho_{0}} v \\
& \frac{\partial \theta}{\partial t}+u \frac{\partial \theta}{\partial x}+v \frac{\partial \theta}{\partial y}=\alpha \nabla^{2} \theta
\end{aligned}
$$

The appropriate initial and boundary conditions are

$$
\begin{aligned}
& t=0: u=v=0, \quad \theta=\theta_{0} ; \quad 0 \leq x \leq H, \quad 0 \leq y \leq L \\
& t>0: u=v=0, \quad \theta=\theta_{c} ; \quad y=0 \\
& u=v=0, \quad \theta=\theta_{h} ; \quad y=L \\
& u=v=0, \quad \frac{\partial \theta}{\partial x}=0 ; \quad x=H \\
& \left.\begin{array}{c}
u=0, \quad \frac{\partial \theta}{\partial x}=0 \\
\mu \frac{\partial v}{\partial x}=-\frac{\partial \sigma}{\partial \theta} \frac{\partial \theta}{\partial y}
\end{array}\right\} ; \quad x=0 .
\end{aligned}
$$

The dynamical boundary condition on the upper free surface relates the velocity gradient to the temperature gradient. It represents the balance between shear stress and surface tension gradient at the surface and is responsible for the establishment of thermocapillary flow in the cavity.

The vorticity-stream function formulation of the problem after non-dimensionalization can be written as

where

$$
\begin{aligned}
& \frac{\partial T}{\partial \tau}+U \frac{\partial T}{\partial X}+V \frac{\partial T}{\partial Y}=\frac{1}{P r} \nabla^{2} T, \\
& \frac{\partial \zeta}{\partial \tau}+U \frac{\partial \zeta}{\partial X}+V \frac{\partial \zeta}{\partial Y}=\frac{G r}{2} \frac{\partial T}{\partial Y}+\nabla^{2} \zeta-H a^{2} \frac{\partial V}{\partial X} \\
& \nabla^{2} \Psi=-\zeta, \\
& U=\frac{\partial \Psi}{\partial Y}, \quad V=-\frac{\partial \Psi}{\partial X},
\end{aligned}
$$

$$
\zeta=\frac{\partial V}{\partial X}-\frac{\partial U}{\partial Y}
$$



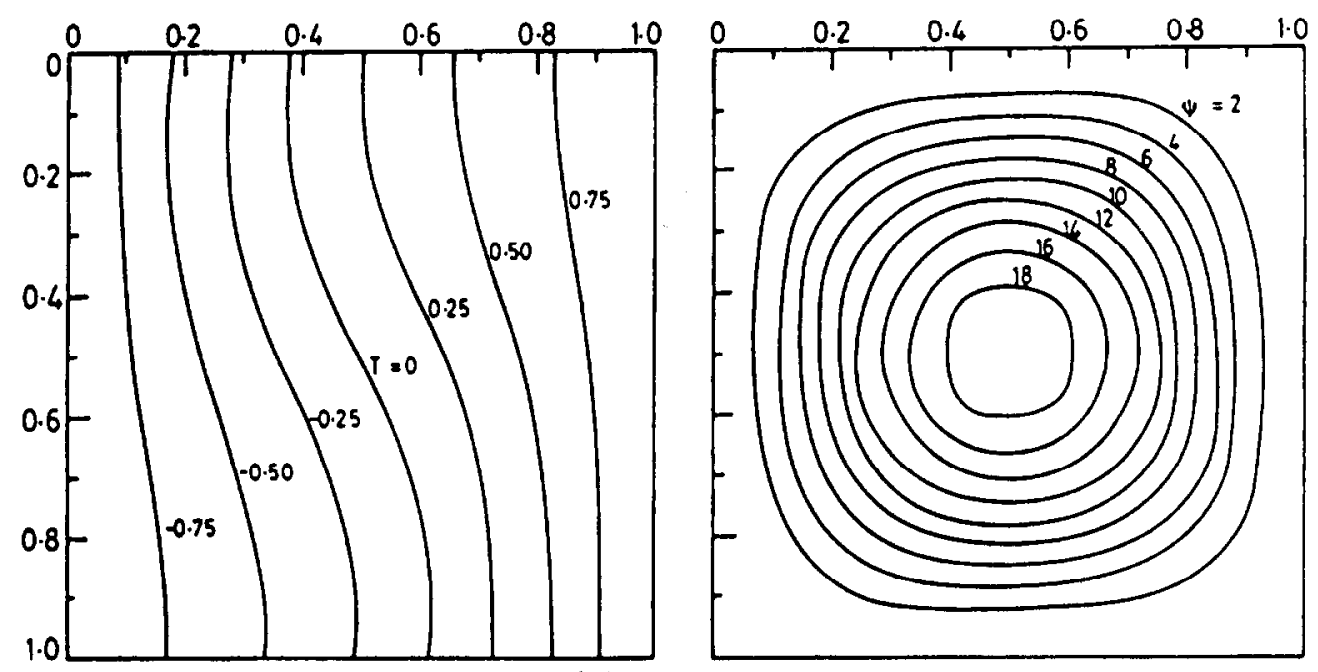

(a) $M a=0$
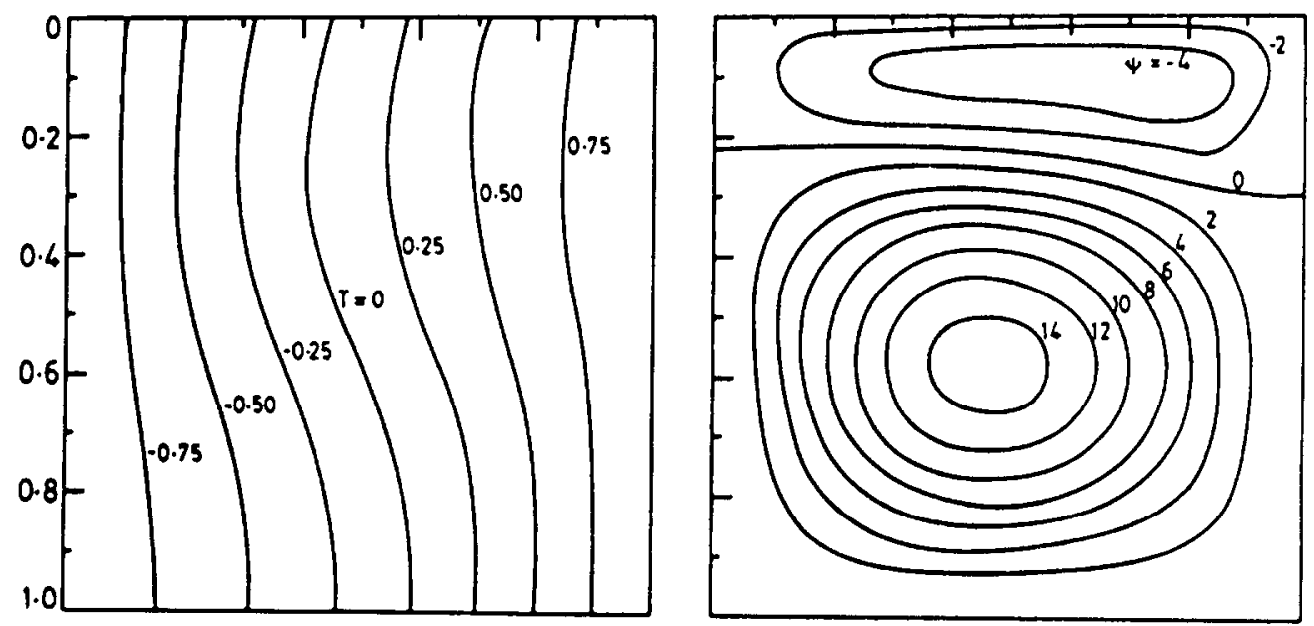

(b) $\mathrm{Ma}=100$

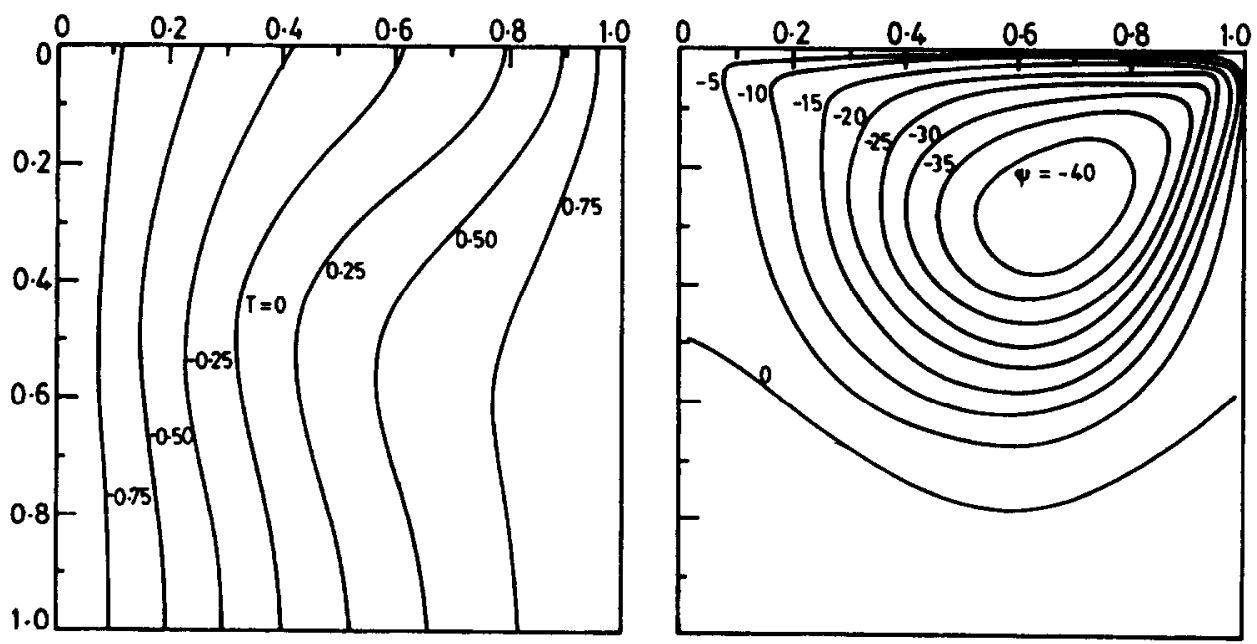

(c) $\mathrm{Ma}=1000$

Fig. 2. Steady-state isotherms and streamlines for $\mathrm{Gr}=2 \times 10^{4}$.

The initial and boundary conditions reduce to

$$
\begin{aligned}
& \tau=0: U=V=\Psi=0, \quad T=\zeta=0 ; \quad 0 \leq X \leq A, \quad 0 \leq Y \leq 1 \\
& \tau>0: \Psi=\frac{\partial \Psi}{\partial Y}=0, \quad T=-1.0 ; \quad Y=0
\end{aligned}
$$




$$
\left.\begin{array}{l}
\Psi=\frac{\partial \Psi}{\partial Y}=0, \quad T=+1.0 ; \quad Y=1 \\
\Psi=\frac{\partial \Psi}{\partial X}=0, \quad \frac{\partial T}{\partial X}=0 ; \quad X=A \\
\Psi=\frac{\partial \Psi}{\partial Y}=0, \quad \frac{\partial T}{\partial X}=0 \\
\zeta=\frac{\partial V}{\partial X}=-\frac{1}{2} \frac{M a}{\operatorname{Pr}} \frac{\partial T}{\partial Y}
\end{array}\right\} ; \quad X=0 .
$$

The above equations are non-dimensionalized using the following variables:

$$
\begin{aligned}
& X=\frac{x}{L}, \quad Y=\frac{y}{L}, \quad U=\frac{u}{(v / L)}, \quad V=\frac{v}{(v / L)} \\
& \tau=\frac{t}{\left(L^{2} / v\right)}, \quad \zeta=\frac{\omega}{\left(v / L^{2}\right)}, \quad \Psi=\frac{\psi}{v} \\
& T=\frac{\theta-\theta_{0}}{\theta_{h}-\theta_{0}} \quad \text { where } \quad \theta_{0}=\frac{\theta_{h}+\theta_{c}}{2} .
\end{aligned}
$$
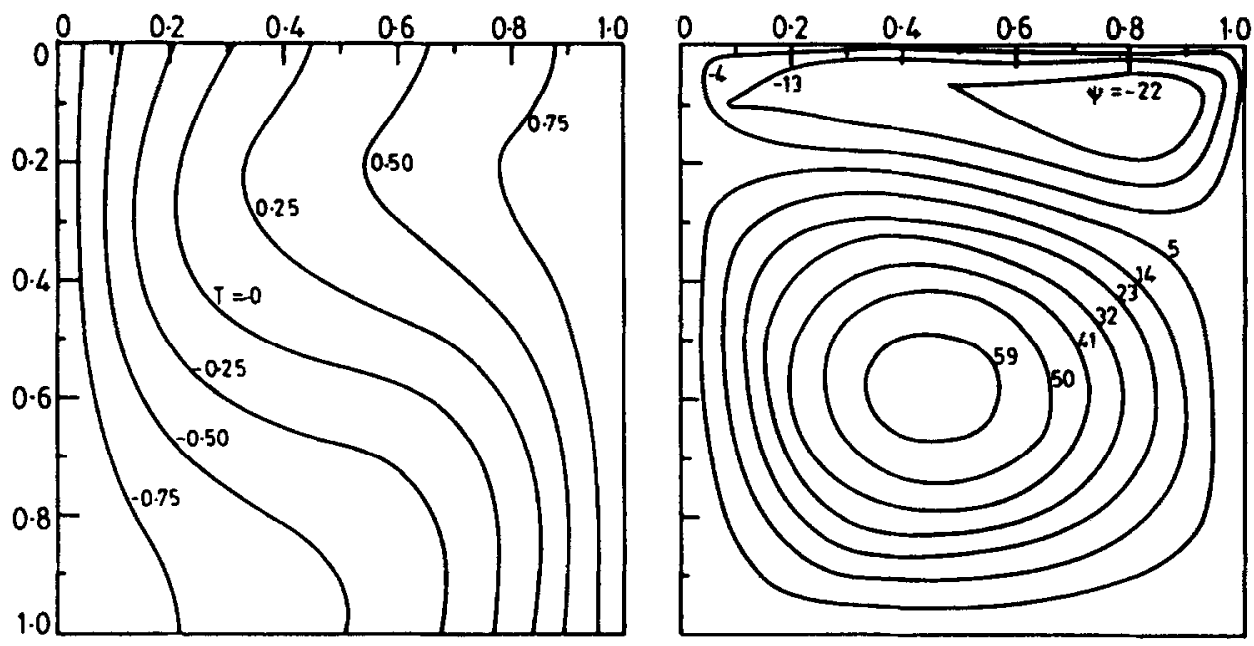

$M a=1000$

Fig. 3. Steady-state isotherms and streamlines for $\mathrm{Gr}=2 \times 10^{5}$.
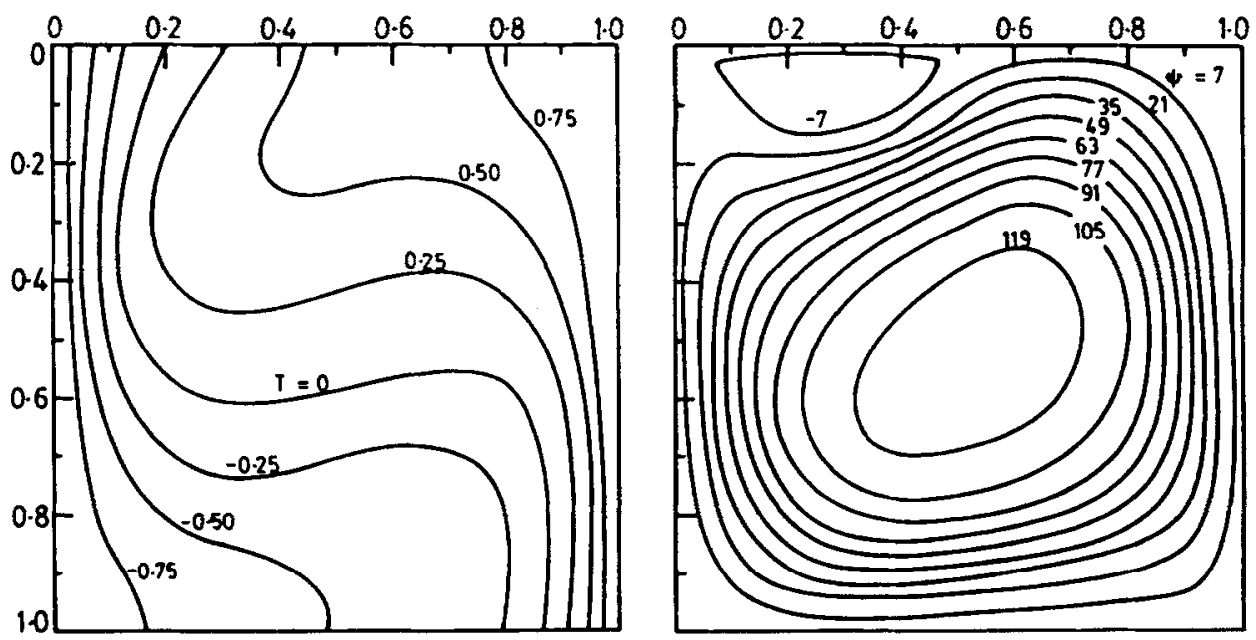

Fig. 4. Steady-state isotherms and streamlines for $G r=2 \times 10^{6}$ and $M a=1000$. 
The non-dimensional parameters are

$\operatorname{Pr}=\frac{\nu}{\alpha}$, the Prandtl number;

$G r=\frac{g \beta_{T}\left(\theta_{h}-\theta_{c}\right) L^{3}}{v^{2}}$, the Grashof number;

$H a=B_{0} L \sqrt{\sigma_{e} / \mu}$, the Hartmann number;

$M a=-\frac{\partial \sigma}{\partial \theta} \frac{\left(\theta_{h}-\theta_{c}\right) L}{\mu \alpha}$, the Marangoni number;

$A=H / L$, the enclosure aspect ratio.

The average Nusselt number, which accounts for the rate of heat transfer through the enclosure, is computed at the lateral walls. The local Nusselt number is defined as

$$
N u=\frac{1}{2}\left(\frac{\partial T}{\partial Y}\right)
$$

The average Nusselt number is expressed as

$$
\overline{N u}=\frac{1}{A} \int_{0}^{A} N u \mathrm{~d} X
$$
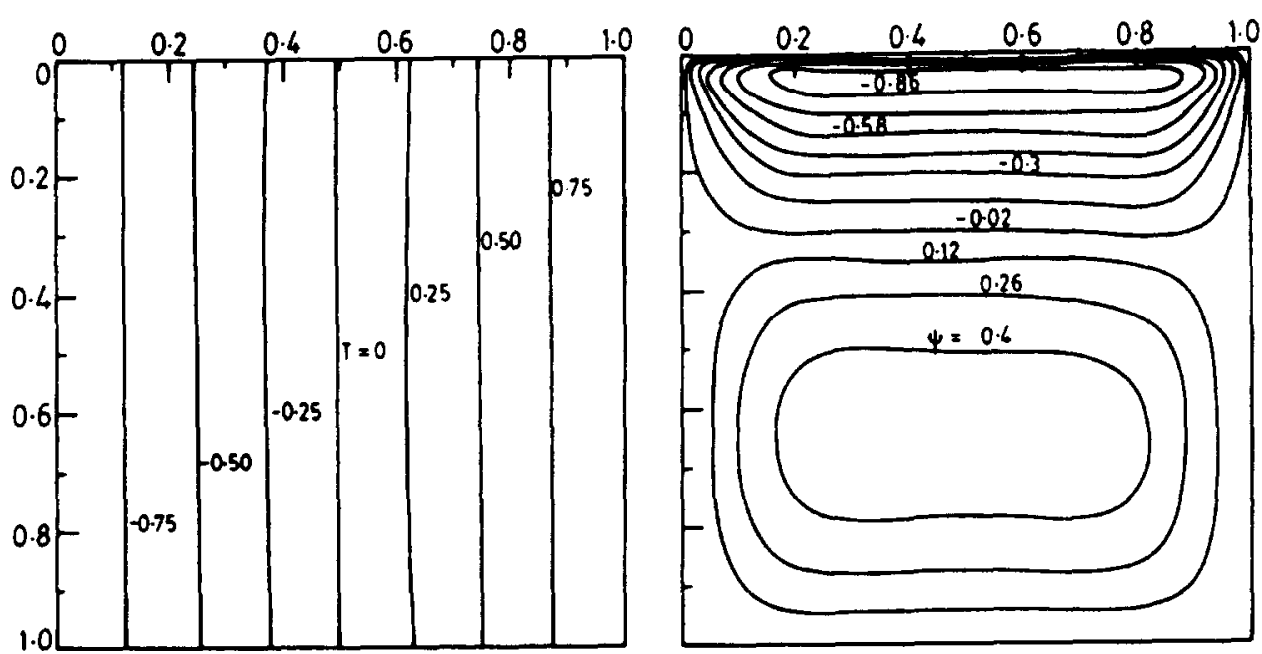

(a) $\mathrm{Ha}=50$
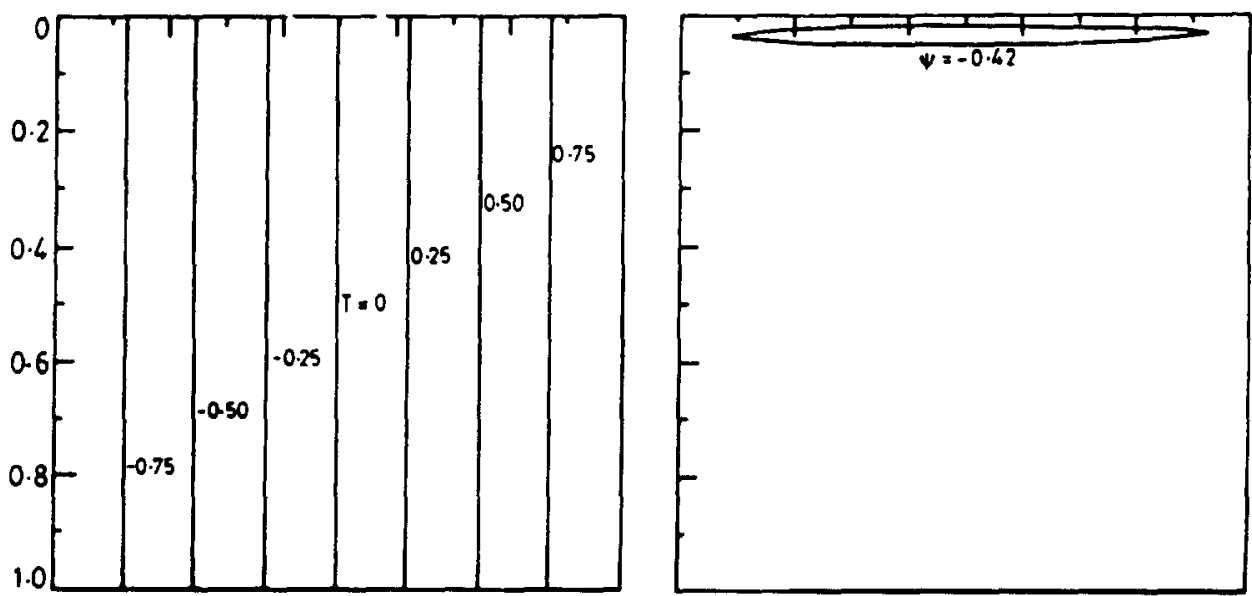

(b) $\mathrm{Ha}_{\mathrm{a}}=100$

Fig. 5(a) and (b). 

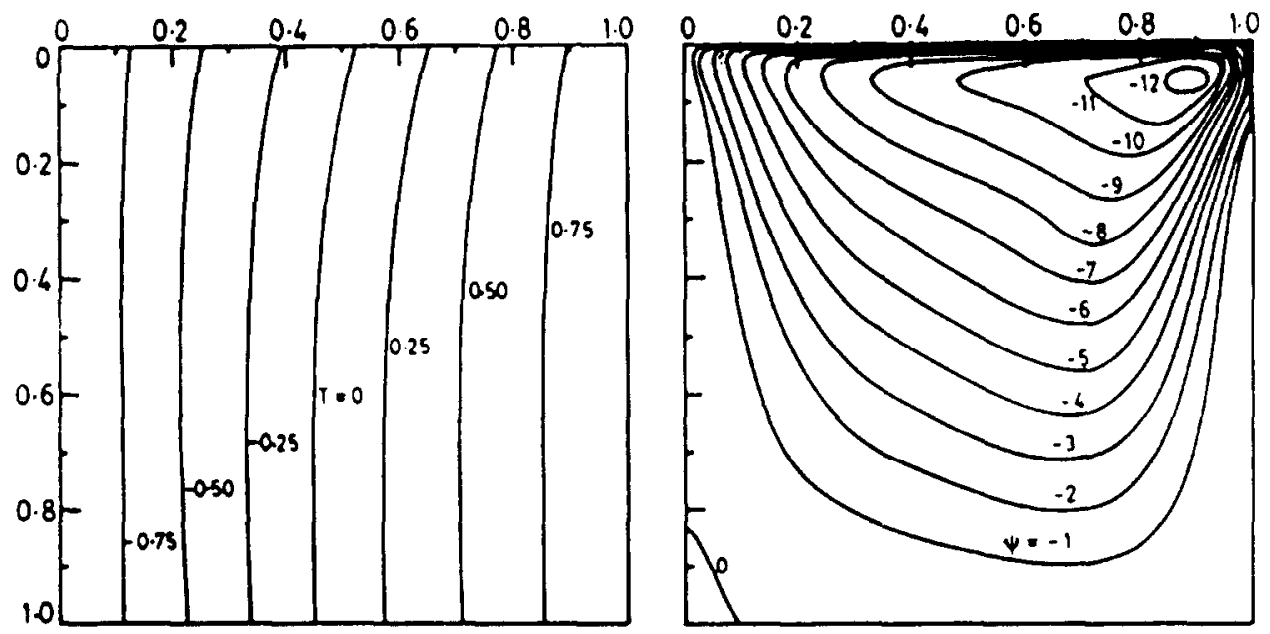

(c) $\mathrm{Ha}=50$
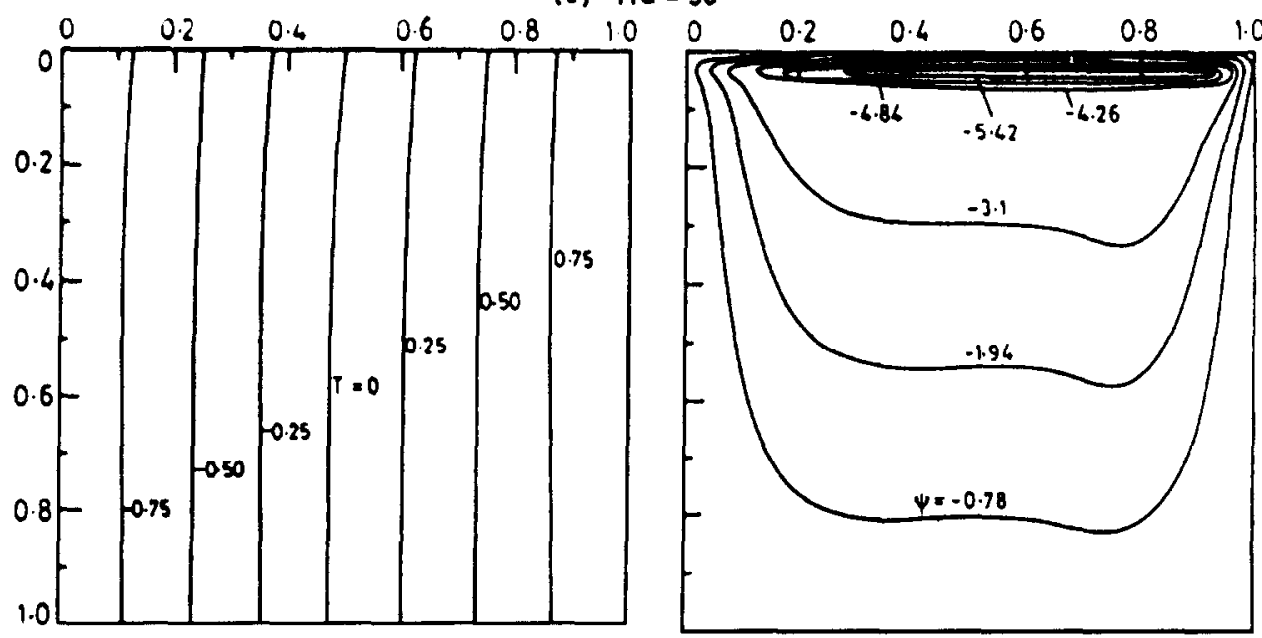

(d) $\mathrm{Ha}=100$

Fig. $5(a)-(d)$. Steady-state isotherms and streamlines for $G r=2 \times 10^{4}$ and $M a=100$

\section{NUMERICAL METHOD}

The equations described in the preceding section are solved numerically using the finite difference technique. Given the field values $T$ and $\zeta$ at any time level, we determine $T$ and $\zeta$ at the next time level from equations (6) and (7), respectively, using the modified ADI (Alternating Direction Implicit) method. The modified two-step ADI method which incorporates the second upwind differencing for non-linear convection-advection terms is used. The central differences are used to approximate the diffusion terms and forward differencing for the time derivatives by retaining the overall second-order accuracy. The stream function is then computed from equation (8) using the newly computed vorticity values. The system of equations obtained from the discretization of equation (8) is solved using the SLOR (Successive Line Over Relaxation) method. Then, the values of $U$ and $V$ at that time level are computed using the central difference approximation to $U=\partial \Psi / \partial Y$ and $V=$ $-\partial \Psi / \partial X$. After obtaining the solution for $T, \zeta$ and $\Psi$ (and hence $U$ and $V$ ) at the first time level, we determine the solutions at the next time levels. The boundary vorticity $\zeta_{b}$ at the solid walls can be derived in the form (see Roache [7])

$$
\zeta_{b}=\frac{\Psi_{b+2}-8 \Psi_{b+1}}{2(\Delta \eta)^{2}}+0(\Delta \eta)^{2}
$$


where $b$ denotes the boundary node and $\Delta \eta$ is the spatial interval in the direction normal to the boundary. However, at the top free surface the vorticity boundary condition is

$$
\zeta_{b}=-\left.\frac{1}{2} \frac{M a}{\operatorname{Pr}} \frac{\partial T}{\partial Y}\right|_{b} .
$$

This computational cycle is then repeated for each of the next time levels and the steady state solution is obtained when the following convergence criteria

$$
\left|\frac{\phi_{i, j}^{n+1}-\phi_{i, j}^{n}}{\phi_{i, j}^{n+1}}\right|<\varepsilon,
$$

for temperature, vorticity and stream function have been met. In the above expression $n$ is any time level, $\varepsilon$ is of order $10^{-5}$ and $\phi$ represents $T, \zeta$ or $\Psi$.

The numerical solution presented here is acquired from a $41 \times 41$ grid system. Prior to the calculations, as a partial verification of the computational procedure, the hydrodynamically developing flow was calculated in the absence of thermocapillary force and magnetic field. The results were compared with the solutions given by Wilkes and Churchill [8] and are found to be in good agreement.

The prominent effects of the governing parameters (i.e. $G r, M a$ and $H a$ ), on the flow structure and the associated heat transfer characteristics, are illustrated in the next section.
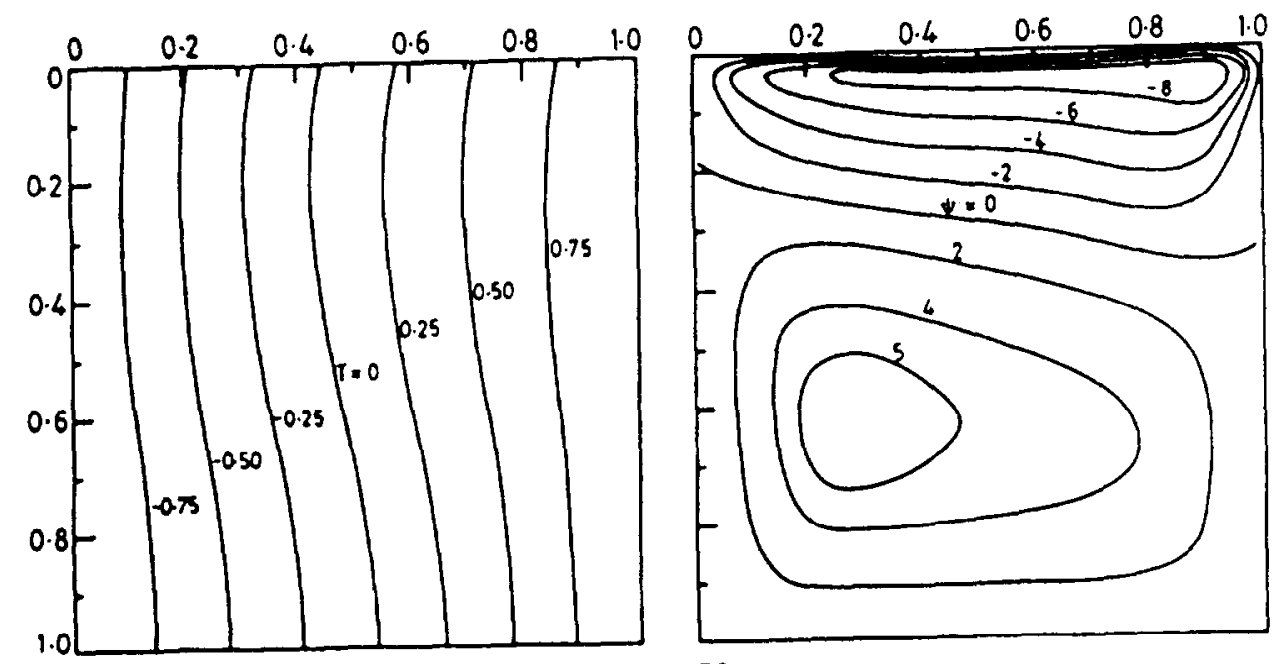

(a) $\mathrm{Ha}=50$
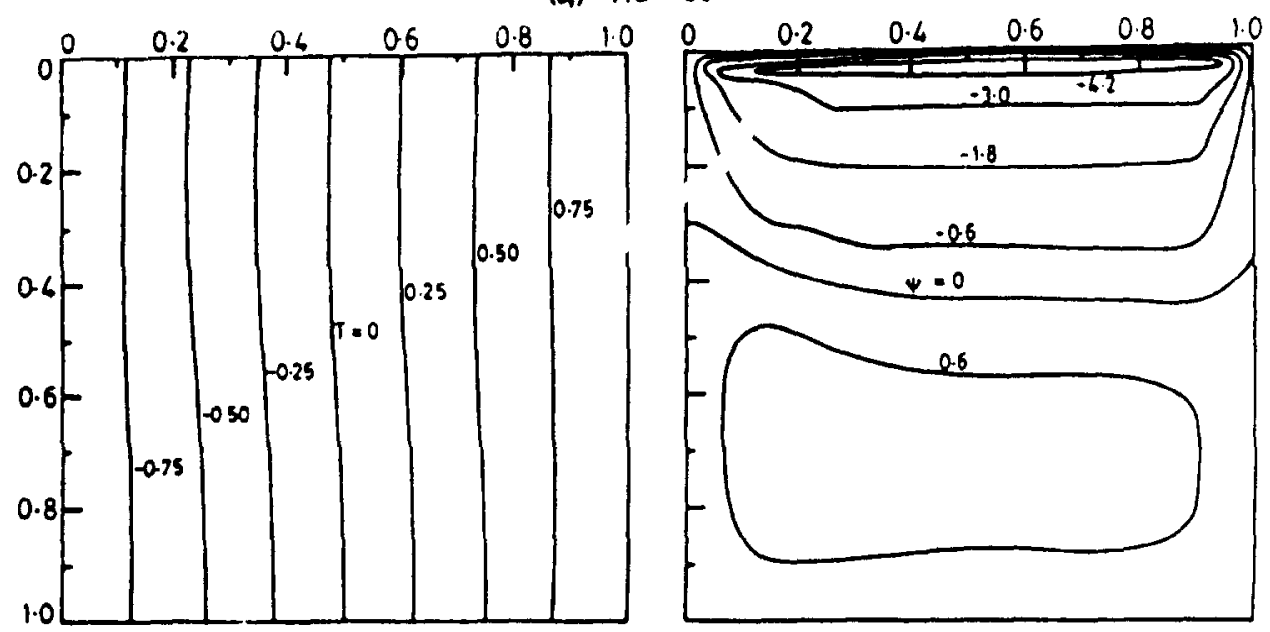

(b) $\mathrm{Ha}=100$

Fig. Steady $\cdots$ therms and streamlines for $G r=2 \times 10^{5}$ and $M a=1000$. 

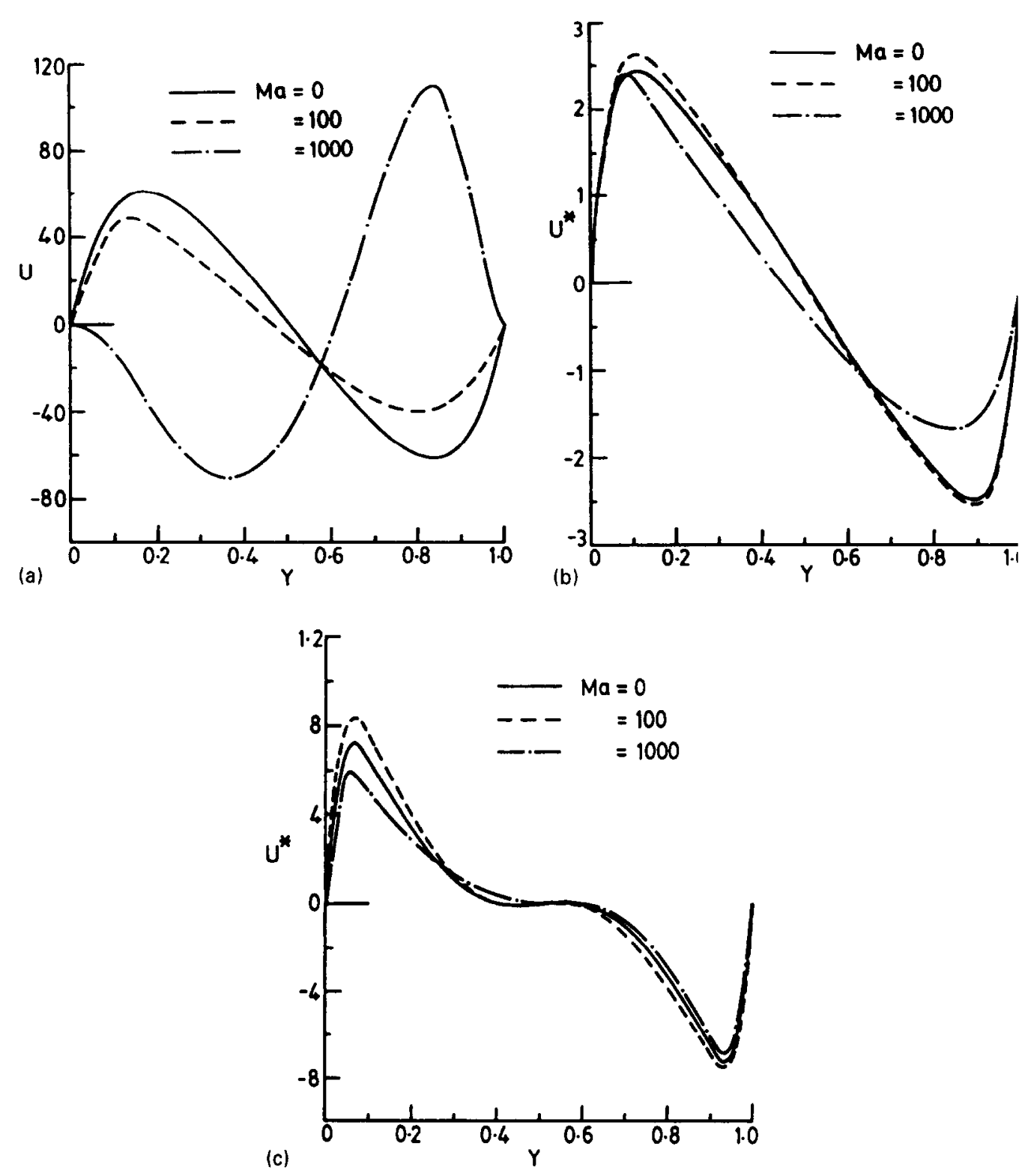

Fig. 7. (a) Vertical velocity at midheight $(X=0.5)$ for $G r=2 \times 10^{4}$. (b) Vertical velocity at midheight ( $X=0.5, U^{*}=U / 100$ ) for $G r=2 \times 10^{5}$. (c) Vertical velocity at midheight $(X=0.5$ and $U^{*}=U / 100$ ) for $G r=2 \times 10^{4}$.

\section{RESULTS AND DISCUSSION}

The effect of magnetic field on the surface tension and buoyancy-induced convection in a rectangular open cavity is investigated numerically. The computations are carried out for a wide range of Grashof number $\left(2 \times 10^{4} \leq G r \leq 2 \times 10^{6}\right)$, Marangoni number $\left(0 \leq M a \leq 10^{5}\right)$ and Hartmann number $(0 \leq H a \leq 100)$ for an enclosure of aspect ratio $A=1$ and Prandtl number $P r$ fixed at 0.054 . The low Prandtl number considered here corresponds to liquid metals. For low-Prandtl number liquids, the ability of the fluid to diffuse heat is much greater than its ability to diffuse vorticity. This property makes the behaviour of these fluids quite distinct. The temperature distribution and flow fields inside the cavity are presented to illustrate the strong influence of magnetic field on the combined mechanism. The rate of heat transfer across the cavity is obtained by evaluating the Nusselt number.

Figure 2 illustrates the streamlines and isotherms at $G r=2 \times 10^{4}$ and $M a=0,100$ and 1000 in the absence of magnetic field. In the absence of shear at the free surface $[\mathrm{Ma}=0$, 
Fig. 2(a)], the flow exhibits a simple recirculating pattern, rising up along the hot wall and sinking along the cold wall of the cavity. The temperature distribution inside the cavity is nearly of conduction type due to the small value of $G r\left(=2 \times 10^{4}\right)$ and the isotherms are nearly antisymmetric. When $M a=100$, surface tension effects lead to the development of a pair of counter-rotating cells at the top and bottom of the cavity. The top cell is driven by thermocapillary forces, while the bottom cell is buoyancy driven [see Fig. 2(b)]. With a further increase in $M a(=1000)$, surface tension induced shear increases the free surface velocities, and consequently streamlines become concentrated in the upper-right corner [see Fig. 2(c)]. This effect is also seen from the temperature distributions, as the isotherms are compacted in the upper-right corner compared to that in the case of $M a=0$ and 100. It is observed that in the moderate Grashof number $\left(G r=2 \times 10^{5}\right)$ and high $G r\left(=2 \times 10^{6}\right)$ regimes, the buoyancy-driven convection predominates for smaller values of $M a(<1000)$. In these cases the isotherms and streamlines are similar to that of pure buoyancy-driven convection. However, for larger values of $M a(\geq 1000)$, counter-rotating cells appear in the enclosure (see Figs 3 and 4).

Figure 5(a) and (b) shows the effect of magnetic field on the combined surface tension and buoyancy-induced convection for $G r=2 \times 10^{4}, M a=100$ and $H a=50$ and 100. The
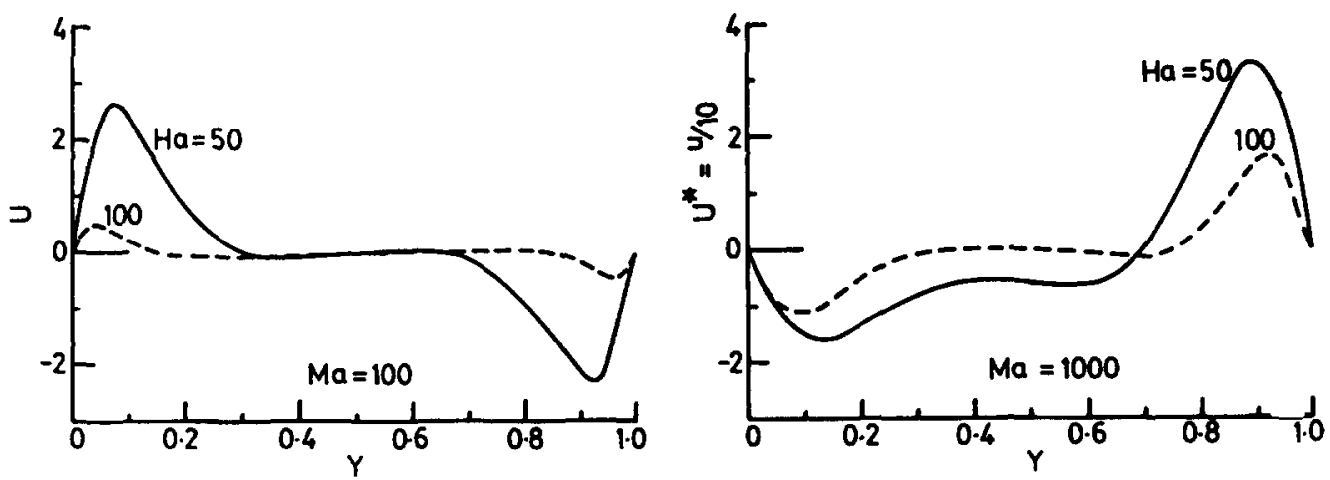

Fig. 8. Vertical velocity profiles at midheight $(X=A / 2)$ for $G r=2 \times 10^{4}$.

Table 1. Effect of $\mathrm{Ma}$ and $\mathrm{Ha}$ on average Nusselt number $(\operatorname{Pr}=0.054, A=1)$

\begin{tabular}{lrrr}
\hline$G r$ & $M a$ & $H a$ & $\overline{N u}$ \\
\hline $2 \times 10^{4}$ & 0 & 0 & 1.11444 \\
& 100 & 0 & 1.11625 \\
& & 50 & 1.01635 \\
& & 100 & 1.00619 \\
& 1000 & 0 & 1.44093 \\
$2 \times 10^{5}$ & & 50 & 1.12676 \\
& & 100 & 1.06831 \\
& 0 & 0 & 1.91219 \\
& 100 & 0 & 1.96543 \\
& & 50 & 1.05367 \\
& & 100 & 1.00885 \\
& 1000 & 0 & 1.97949 \\
& & 50 & 1.13910 \\
& & 100 & 1.06713 \\
& $10^{4}$ & 0 & 2.73628 \\
& & 0 & 3.40480 \\
& 100 & 0 & 3.48475 \\
& & 50 & 2.25035 \\
& & 100 & 1.26864 \\
& & 0 & 3.65707 \\
& 1000 & 50 & 2.33030 \\
& & 0 & 1.33220 \\
& & & 4.09708 \\
\hline & & &
\end{tabular}



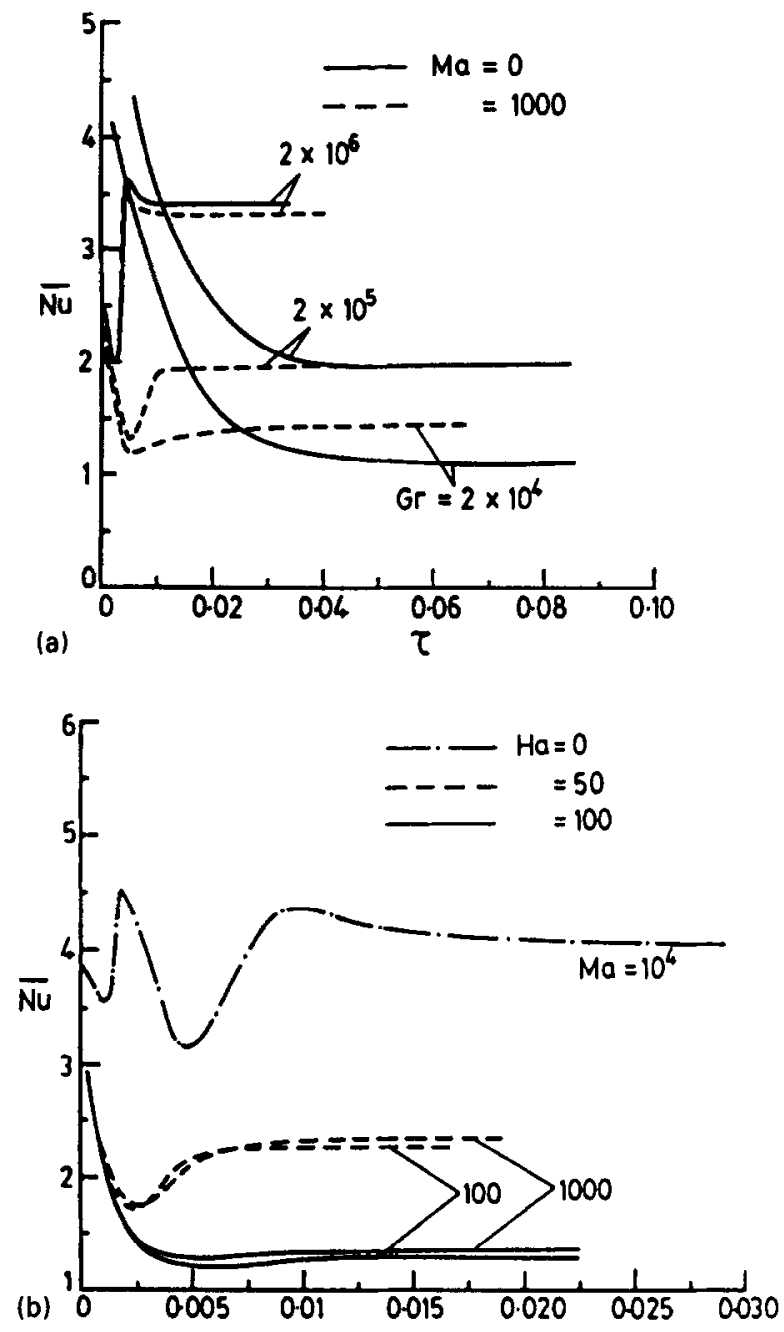

Fig. 9. (a) Average Nusselt number vs time $\tau$. (b) Average Nusselt number vs time for $G r=2 \times 10^{6}$.

isotherms become almost parallel at equal distance, resembling the conduction state due to the presence of magnetic field. The fluid flow in the cavity is established by a relatively balanced interaction of the two driving mechanisms involved, with a strong dependence on $H a$. The balance of the shear and buoyancy effects is manifested in the formation of two eddies, as shown in Fig. 5(a). The streamlines are elongated in nature and the upper cell is crowded and elongated along the free surface. The increase in the magnetic field strength $(H a=100)$ does not have much impact on the temperature field. However, the flow field is drastically changed with a single eddy at the top of the cavity, which is stretched along the free surface. This shows that convection is completely suppressed due to the strong magnetic field effect and the heat transfer in the cavity is mostly due-to conduction alone [see Fig. 5(b)].

As the surface tension force increases further $(M a=1000)$ and for $H a=50$, the isotherms and streamline pattern are depicted in Fig. 5(c). There is a slight change in isotherms and they are slightly pushed to the cold wall side. The streamlines are concentrated near the right corner of the free surface accompanied by a corner eddy at the bottom-left corner. The temperature is distorted from a linear conductive field. For $\mathrm{Ha}=100$ [Fig. 5(d)], the isotherms become almost parallel and the streamlines are still concentrated and also elongated at the top free surface. Figure 6 depicts the effect of magnetic field when $G r=2 \times 10^{5}$ and $M a=1000$. When the Grashof number is high and magnetic field is small [Fig. 6(a)], the buoyancy-driven convection is the dominant mechanism. As magnetic field strength increases [Fig. 6(b)], the buoyancy-driven convection is reduced. 
Figures 7(a)-(c) and 8 show the vertical velocity profiles at the midheight of the enclosure $(X=A / 2)$ for different values of $G r, M a$ and $H a$. The effect of $M a$ and $H a$ on the flow is more evident from these profiles, as they change substantially when $M a$ and $H a$ are changed.

Table 1 lists the average Nusselt number $\overline{N u}$ for various combination of $\mathrm{Gr}, \mathrm{Ma}$ and $\mathrm{Ha}$. From this table we obscrve that $\overline{N u}$ increases with $M a$ and decreases with $\mathrm{Ha}$. Figure 9 shows the variation of average Nusselt number with time $\tau$ for various values of $G r, M a$ and $\mathrm{Ha}$. At early stages of the flow development, the heat transfer is due mainly to pure conduction. During the transition from a conduction- to a convection-dominated heat transfer mode, the Nusselt number curves pass through a minimum. The average Nusselt number then increases slightly and levels off to reach the steady state [see Fig. 9(a)]. From Fig. 9(b) we observe an overshooting of the cavity wall Nusselt number at $M a=10^{4}$ and $H a=0$, at the initial time, which then dampens to reach the steady state. These oscillations in the time history of Nusselt numbers are due to the existence of thermal instabilities caused by the two driving mechanisms acting on the flow.

\section{REFERENCES}

1. T. L. Bergman and S. Ramadhyani, Combined buoyancy and thermocapillary driven convection in open square cavities. Numer. Heat Transfer 9, 441 (1986).

2. J. Srinivasan and B. Basu, A numerical study of thermocapillary flow in a rectangular cavity during laser melting. Int. J. Heat Mass Transfer 29, 563 (1986).

3. B. Basu and J. Srinivasan, Numerical study of steady-state laser melting problem. Int. J. Heat Mass Transfer 31, 2331 (1988).

4. J. C. Chen and Y. C. Huang, Thermocapillary flows of surface melting due to a moving heat flux. Int. J. Heat Mass Transfer 34, 663 (1990).

5. B. M. Carpenter and G. M. Homsy, Combined buoyant thermocapillary flow in a cavity. J. Fluid Mech. 207, 121 (1989).

6. H. Ben Hadid and B. Roux, Buoyancy and thermocapillary driven flows in differentially heated cavities for low-Prandtl number fluids. J. Fluid Mech. 235, 1 (1992).

7. P. J. Roache, Computational Fluid Dynamics. Hermosa, Albuquerque (1976).

8. J. O. Wilkes and S. W. Churchill, The finite-difference computation of natural convection in a rectangular enclosure. A.I.Ch.E. J. 12, 161 (1966). 